\title{
The Effect of Noise in Images on Electron Tomography Reconstruction
}

\author{
Misa Hayashida ${ }^{1}$, Michael Bergen ${ }^{2}$, Peng $\operatorname{Li}^{2}$, Tomohiko Iijima ${ }^{1}$, Shinichi Ogawa ${ }^{1}$, Marek Malac ${ }^{2,3}$ \\ 1. National Institute of Advanced Industrial Science and Technology (AIST), Ibaraki, Japan \\ 2. National Institute of Nanotechnology, Edmonton, AB, Canada \\ 3. Department of Physics, University of Alberta, Edmonton, AB, Canada
}

Electron tomography (ET) allows to study morphology of nanoscale objects in three dimensions (3D) and can provide practical insights in origin of material properties at nanoscale. To obtain 3D reconstructed volume, a series of images needs to be acquired at small $\left(2^{\circ}\right.$ to $\left.5^{\circ}\right)$ increments over at least $\pm 60^{\circ}$ angular range. This requirement corresponds to 60 to 90 images that need to be collected from the same area of a sample. The large number of images translates to a high electron irradiation dose and consequent damage preventing for example investigation of biological samples at resolution below 1 $\mathrm{nm}$. Although it is necessary to reduce electron dose as possible as to reduce the damage, it is necessary to acquire sufficient counting statistics to obtain $3 \mathrm{D}$ reconstructed images with signal to noise ratio (SNR) $>3$ (the Rose criterion). To reduce the overall irradiation dose individual images of the tilt series should be acquired with the lowest dose that provides acceptable SNR of the reconstructed volume. We report on noise propagation from the individual images to the reconstructed volume and provide practical guidance on acquisition conditions of the tilt series.

Moreover, to acquire accurate 3D images, precise alignment of the tilt series is important before reconstruction. A fiducial marker method is a typical method for carrying out alignments [1]. The accuracy of detected markers' position effects on image quality of 3D images. The accuracy becomes lower if SNR of individual images in a tilt series become more less because of noise. We report the dependence of accuracy of the fiducial markers' position detection on the electron irradiation dose of the individual images.

A rod-shaped test sample, containing gold nano-particles embedded in silica was imaged in a Hitachi HF 3300 , a $300 \mathrm{kV}$ TEM/STEM equipped with a cold field emission gun. The tilt angle step was $3^{\circ}$ and angular range was $\pm 90^{\circ}$ avoiding the missing wedge effect. At each tilt angle images were acquired with $0.1,0.51,3$ and $5 \mathrm{~s}$ acquisition time corresponding to approximately $13,66,130,660 \mathrm{e} / \AA^{2}$ per image with pixel size $1.2 \AA^{2}$. The detectability of the Au particles as function of dose was evaluated by examining a histogram of the reconstructed volume. A second sample, composed of fiducial markers on carbon rod, was examined under same conditions and the error of detected position of the markers was evaluated as function of irradiation dose per image. The tungsten fiducial markers were fabricated using a Helium ion microscope (HIM) [2]. Images were collected 0.1, 0.3, 0.5, 1 and $3 \mathrm{~s}$ acquisition time corresponding to approximately $2,6,10,20,62 \mathrm{e} / \AA^{2}$ respectively in TEM mode. Annular dark field scanning TEM (STEM) performance was also evaluated. The ET volume was reconstructed using standard filtered back projection (FBP) method.

Fig. 1a shows a cross section of the reconstructed volume of the Au in silica sample for various incident dose (i.e. image acquisition time). The images were binned from 2048 to 512 pixels prior reconstruction increasing the counting statistics per pixel, but not the irradiation dose at the sample plane. The shape of the Gatan Ultrascan $1000^{\mathrm{TM}}$ camera modulation transfer function suggests only minor effect on the resolution as a result of such binning. Fig. 1b shows example histograms of the reconstructed 3D 
volumes shown in Fig. 1a.

Fig. 2a shows an image of the test sample with HIM markers. It was also imaged in both of TEM and STEM mode by the same microscope. The diameter of the rod approximately $250 \mathrm{~nm}$ and marker diameter is less than $10 \mathrm{~nm}$. At each tilt angle of the tilt series the markers were detected by using an automatic method [3] that is based on template matching. In fiducial marker method, minimum of the root mean square error between expected markers' positions in the specimen and the positions in images is sought. We used the minimum of the root mean square error as error of detected position of the markers. Fig. $2 \mathrm{~b}$ shows the relationship between the error and electron dose of individual images. Fig. 1a shows that about $0.5 \mathrm{~s}$ images show nearly the same detectability of Au particles as $1 \mathrm{~s}$ and $5 \mathrm{~s}$ images translating to $66 \mathrm{e} / \AA^{2}$ per image is needed to reliably detect the markers. The Fig. $2 \mathrm{~b}$ indicates that little is gained in terms of the error with dose exceeding about $0.3 \mathrm{~s}$, or $6 \mathrm{e} / \AA^{2}$ per image.

\section{References:}

[1] MC Lawrence, in: Frank, J. (Ed.), Electron Tomography. Plenum Press, New York, (1992) 197.

[2] M Hayashida, T Iijima, T Fujimoto and S Ogawa, Micron 43 (2012), 992.

[3] M. Hayashida et. al, (Submitted).

[4] The sample was kindly provided by X. Wang and R. Lockwood. Discussions with Robb A. McLeod provided insight in practical evaluation of level of noise in images. Support from NINT/NRC (Canada) and AIST (Japan) is gratefully acknowledged.

(a)
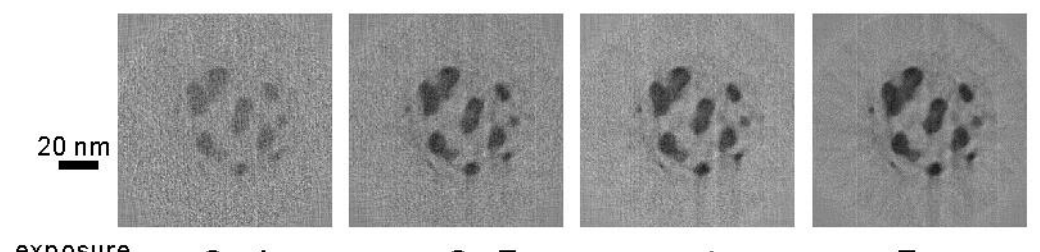

exposure
time(sec) $\quad 0.1$

0.5

1

5

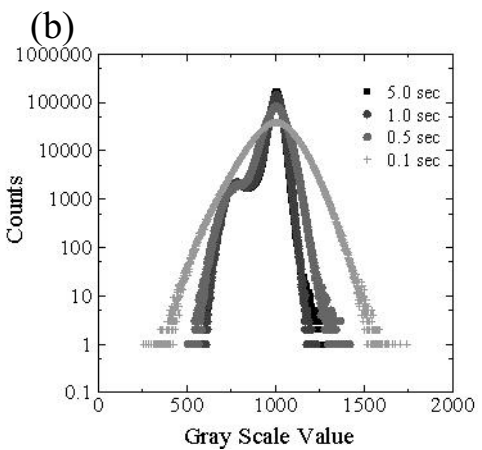

Figure 1. a) Cross section from a reconstructed 3D volume for image acquisition time $0.1 \mathrm{~s}$ to $5 \mathrm{~s}$. Qualitatively the ability to detect object increases with increasing acquisition time as a result of improved counting statistics. b) Histograms of the reconstructed 3D volumes.

(a)

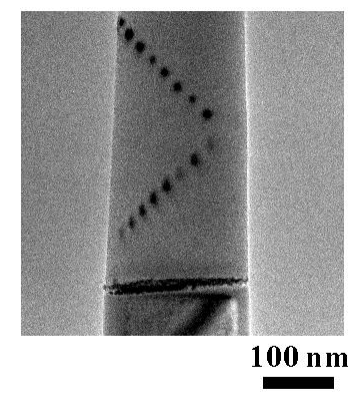

(b)

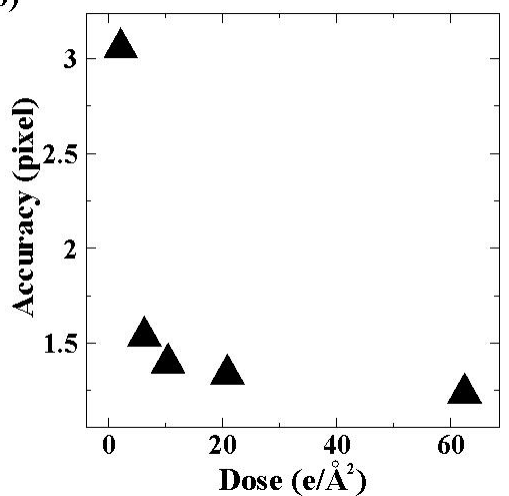

Figure 2. a) TEM image of carbon rod with markers deposited by HIM. b) Relationship between the error of detected position of the markers and electron dose of individual images. 